\title{
Review \\ Beyond antibiotics in severe community-acquired pneumonia: the role and rationale for tissue factor pathway inhibition
}

\author{
Pierre-Francois Laterre
}

St Luc University Hospital, Université Catholique de Louvain, UCL, 1200 Brussels, Belgium

Corresponding author: Pierre-Francois Laterre, Pierre-Francois.Laterre@uclouvain.be

Published: 26 November 2008

This article is online at http://ccforum.com/content/12/S6/S4

(c) 2008 BioMed Central Ltd
Critical Care 2008, 12(Suppl 6):S4 (doi:10.1186/cc7027)

thrombin formation and proinflammatory intracellular signaling, in which protease-activated receptor (PAR)-1 and PAR-2 are involved. It is likely that over-expression of TF, both systemically and in the lung, contributes to the pathophysiology of severe community-acquired pneumonia (CAP) $[3,4]$, which is associated with a 28-day all-cause mortality in excess of $30 \%[5,6]$.

TF pathway inhibitor (TFPI) is an endogenous molecule with both anti-inflammatory and anti-coagulant activities (Figure 1). However, in severe infection endogenous TFPI is overwhelmed by increased expression of TF [7]. Data from both animal models and from the clinic suggest that, in this setting, administration of recombinant TFPI (tifacogin) can reduce acute lung injury and improve prospects for survival.

\section{From laboratory to clinic}

In a baboon model of potentially lethal septic shock induced by intravenous infusion of Escherichia coli, administration of recombinant TFPI $6 \mathrm{mg} / \mathrm{kg}$ significantly attenuated the coagulation response, decreased damage to target organs, including the lung, and reduced mortality [8]. In a rat model of lung injury induced by lipopolysaccharide (LPS), treatment with recombinant TFPI before and after the insult reduced vascular permeability, edema, neutrophil infiltration, and production of tumor necrosis factor- $\alpha$ by stimulated monocytes [9].

The role played by the TF-FVIla complex in acute lung injury has been confirmed by work conducted in an E. coli baboon model, which showed that both TFPI and site-inactivated FVIla (a competitive inhibitor of TF) have protective effects [4]. Site-inactivated FVIla has also been shown to reduce local release of proinflammatory cytokines, preserve gas exchange, and reduce fibrin deposition and lung edema following intratracheal administration of LPS in rats [10]. It

CNS = central nervous system; FVIla = activated factor VII; ICU = intensive care unit; IL = interleukin; INR = international normalized ratio; LPS = lipopolysaccharide; OPTIMIST = Optimized Phase 3 Tifacogin in Multicenter International Sepsis Trial; PAR = protease-activated receptor; PF = prothrombin fragment; TF = tissue factor; TFPI = tissue factor pathway inhibitor. 


\section{Figure 1}

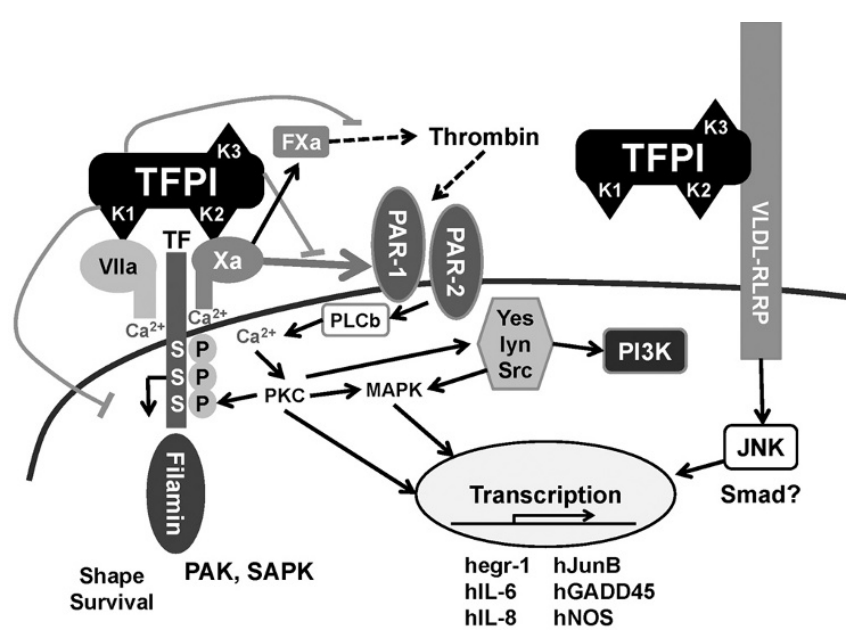

TFPI anti-coagulant and anti-inflammatory activities. TFPI limits the conversion by the TF-FVIla complex from factor $\mathrm{X}$ to $\mathrm{Xa}$ and thrombin formation, and thereby reduces proinflammatory intracellular signaling via PAR-1 and PAR-2 receptors. TFPI attaches the LPS-binding protein complex and alters the host responses to bacteria through interaction with TLRs and CD14. hegr-1, human early growth response protein-1; hGADD45, growth arrest and DNA damage inducible gene; hIL-6, human interleukin-6; hIL-8, human interleukin-8; hJunB, oncogene; hNOS, human nitric oxide synthase; JNK, Jun aminoterminal kinase; LPS, lipopolysaccharide; lyn, oncogene homolog, Src; MAPK, mitogen-activated protein kinases; PAK, p21-activated protein kinase; PAR, protease-activated receptor; PI3K, phosphatidylinositol-3kinase; PKC, protein kinase C; PLCb, phospholipase CB; SAPK, stress-activated protein kinase; Smad, moderates activity of TGF- $\beta$ ligands; TF, tissue factor; TFPI, tissue factor pathway inhibitor; TLR, Toll-like receptor; Yes, a tyrosine protein kinase.

has been established that TFPI is able to attach the LPSbinding protein complex [11], altering the host response to bacteria through interaction with Toll-like receptors and CD14.

These laboratory findings formed the rationale for the initial investigation of recombinant TFPI in the clinic. In 2001, a randomized, controlled Phase 2 trial conducted in 210 patients with severe sepsis provided evidence of a survival benefit [12]. Mortality among patients given tifacogin (at doses of 0.025 or $0.05 \mathrm{mg} / \mathrm{kg}$ per hour infused over 96 hours) was $30 \%$. This was appreciably lower than the $38 \%$ rate of mortality seen among patients in the placebo group (not significant). Logistic regression analysis suggested that greater activation of coagulation at baseline, indicated by a higher international normalized ratio (INR), was associated with a more pronounced benefit from tifacogin. Evidence of biological activity of tifacogin was demonstrated by reduced formation of thrombin and thrombin-antithrombin complex, and lower levels of the inflammatory mediator IL-6. No excess of bleeding events was observed in the tifacogin-treated group. These encouraging findings led to the design of the initial Phase 3 trial, described below.

\section{Study TFP007: OPTIMIST}

The Phase 3 trial OPTIMIST (Optimized Phase 3 Tifacogin in Multicenter International Sepsis Trial) [13] included 1,754 patients, who were randomly assigned to either placebo or tifacogin, given as an infusion of $0.025 \mathrm{mg} / \mathrm{kg}$ per hour over a period of 96 hours. Patients included had at least two dysfunctional organs and an INR of 1.2 or greater. The two groups were well matched in terms of mean age (62 years in both groups), baseline Acute Physiology and Chronic Health Evaluation (APACHE) II score (25 in both groups), and organ dysfunction (3.1 in the placebo group, 3.0 among tifacogintreated patients). The two arms of the study were also closely matched in terms of other relevant variables. The overall 28day mortality rates in the tifacogin and placebo groups were $34.2 \%$ and $33.9 \%(P=0.75)$, respectively, after adjustment per protocol for baseline APACHE II score and IL-6.

A second, smaller group of 201 patients with a less severe coagulation abnormality was subsequently enrolled as a safety cohort. Among patients in this secondary cohort, who all had an INR of less than 1.2, the 28-day mortality rate among those who received tifacogin was significantly lower than that in the placebo group $(12.0 \%$ versus $22.9 \%$; $P=0.025)$. Although overall the trial was unable to identify a survival benefit for tifacogin, this finding suggested that tifacogin might have greater efficacy among subgroups of patients. It was also hypothesized that the explanation for the negative results might lie in the fact that heparin prophylaxis for deep vein thrombosis had been permitted in the main study [13].

\section{Efficacy in subgroups defined by heparin exposure and documentation of infection by culture}

There is a biological interaction between TFPI and heparin [14]. TFPI exhibits competitive low-affinity binding with glycosoaminoglycans, and heparin causes release of endogenous TFPI from its binding sites on endothelium. The majority of patients in the study received heparin prophylaxis during or after study drug infusion (either unfractionated heparin or lowmolecular-weight heparin). Heparin prophylaxis was left to the discretion of the clinician, and randomization was not stratified according to heparin use.

In these patients there may have been inadequate interaction between tifacogin and the TF/FVIla complex, resulting in reduced efficacy of TFPI. Subgroup analyses were therefore undertaken to distinguish between patients who had received any dose of heparin, with the exception of heparin flushes for arterial lines, and those who had not. Because the type of heparin used was highly variable at each investigator site, no analysis was performed to correlate heparin dose with clinical or biological responses. Analyses were conducted separately for patients with levels of circulating prothrombin fragment (PF) 1.2 of $3 \mathrm{ng} / \mathrm{ml}$ or greater (which reflects pronounced activation of coagulation) and those with PF1.2 levels of less than $3 \mathrm{ng} / \mathrm{ml}$. Among the 238 patients with greater activation 


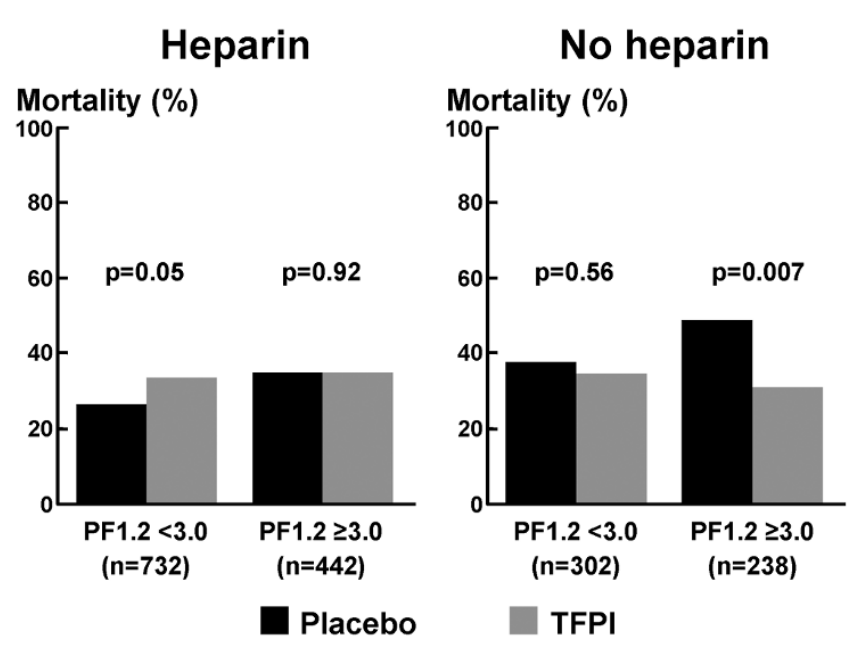

28-Day mortality: heparin versus no heparin. Shown are 28-day all-cause mortality rates in patients with or without heparin prophylaxis during the 4-day study drug infusion according to prothrombin fragment (PF)1.2 plasma levels. TFPI, tissue factor pathway inhibitor.

of coagulation who did not receive heparin, there was evidence of a significant benefit from tifacogin. Mortality in the placebo group was approximately $50 \%$, whereas that in the tifacogin-treated group was approximately $30 \%(P=0.007$; Figure 2). No such effect was observed among patients not receiving heparin patients who exhibited less pronounced activation of coagulation, and neither was this effect observed among patients who had received heparin.

Many patients enrolled in sepsis trials have suspected infection that is not subsequently documented. If at least part of the mechanism of action of tifacogin is through modulation of the host's antibacterial response, then the presence of infection may influence the efficacy of tifacogin.

Aspects of inflammatory signaling relate to innate mechanisms of immunity. TFPI is known to have relevant effects such as LPS binding and inhibition of the interaction of the LPS/LPS-binding protein complex with CD-14 and Toll-like receptor-4 [11].

Investigators therefore examined outcome by culture status. Independent of heparin exposure, patients in the primary cohort (INR $\geq 1.2$ ) who had infection documented by positive blood culture exhibited a strong trend toward reduced mortality when they were given tifacogin rather than placebo (mortality rate $27.7 \%$ versus $35.4 \% ; P=0.054$ ). There was no sign of survival benefit among patients with positive evidence of infection other than blood culture. Also, among patients who had a negative culture or whose infection status was not determined, there was evidence that the tifacogin
Table 1

28-Day all-cause mortality in TFPI-treated and placebo-treated patients

\begin{tabular}{llll}
\hline & \multicolumn{3}{c}{ Overall } \\
\cline { 2 - 4 } INR $\geq 1.2$ & Placebo & TFPI & $P$ \\
\hline Positive blood culture & & & \\
$\quad n$ & 257 & 278 & 0.054 \\
$\quad$ Total (\%) & 29.4 & 31.6 & \\
$\quad$ Mortality (\%) & 35.4 & 27.7 & \\
Positive other & & & \\
$\quad n$ & 387 & 370 & \\
$\quad$ Total (\%) & 44.3 & 42.0 & \\
$\quad$ Mortality (\%) & 35.1 & 35.4 & \\
Negative culture/not done & & & 0.02 \\
$\quad n$ & 230 & 232 & \\
$\quad$ Total (\%) & 26.3 & 26.4 & \\
$\quad$ Mortality (\%) & 30.0 & 40.1 & \\
\hline
\end{tabular}

Presented are 28-day all-cause mortality rates in TFPI and placebotreated patients with positive blood cultures, other documented infections, and negative culture or undocumented infections. INR, international normalized ratio; TFPI, Tissue factor pathway inhibitor.

group fared worse (mortality rate $40.1 \%$ versus $30.0 \%$ in the placebo group; $P=0.02$; Table 1 ).

The suggestion of mortality benefit among patients with positive blood culture was stronger still when only those patients not exposed to heparin were included in the subgroup analysis. In this instance, the mortality among placebo patients was $40.0 \%$ but only $26.9 \%$ among those who received tifacogin ( $P=0.05$; Table 2$)$.

\section{Bleeding or thrombotic events}

Overall, the incidence of bleeding events (during infusion or within 2 days) was similar in the tifacogin and placebo groups (4\% versus 3\%). The risk for any thromboembolic event over the 28 day study period was identical (at 3\%) in the two arms of the study. This rate of $3 \%$ was the same in patients exposed to tifacogin alone and in patients who had also been treated with heparin. This finding does not imply that heparin prophylaxis is not mandatory in critically ill patients, but it provides certain reassurance that the current randomized trial of tifacogin versus placebo (see the section on CAPTIVATE, below) is justified. Indeed, patients who were not receiving heparin in the OPTIMIST trial had more pronounced coagulation disturbances and lower platelet count, and were therefore less likely to require prophylaxis against deep vein thrombosis.

No placebo patient experienced a central nervous system (CNS) bleed. This compares with five cases among tifacogin 
Table 2

28-Day all-cause mortality in patients with and those without heparin prophylaxis

\begin{tabular}{|c|c|c|c|c|c|c|}
\hline \multirow[b]{2}{*}{ INR $\geq 1.2$} & \multicolumn{3}{|c|}{ Any heparin } & \multicolumn{3}{|c|}{ No heparin } \\
\hline & Placebo & TFPI & $P$ & Placebo & TFPI & $P$ \\
\hline \multicolumn{7}{|c|}{ Positive blood culture } \\
\hline$n$ & 162 & 174 & 0.31 & 95 & 104 & 0.05 \\
\hline Mortality (\%) & 32.7 & 28.2 & & 40.0 & 26.9 & \\
\hline \multicolumn{7}{|l|}{ Positive other } \\
\hline$n$ & 271 & 267 & 0.39 & 116 & 103 & 0.24 \\
\hline Mortality (\%) & 30.6 & 34.5 & & 45.7 & 37.9 & \\
\hline \multicolumn{7}{|c|}{ Negative culture/not done } \\
\hline$n$ & 167 & 159 & 0.01 & 63 & 73 & 0.98 \\
\hline Mortality (\%) & 25.7 & 39.6 & & 41.3 & 41.1 & \\
\hline
\end{tabular}

Presented are 28-day all-cause mortality rates in patients with or without heparin prophylaxis during the study drug infusion period and with positive blood cultures, other documented infections, and negative culture or undocumented infections. INR, international normalized ratio; TFPI, Tissue factor pathway inhibitor.

patients, but this represents an incidence of less than $1 \%$. Moreover, the increased risk for a hemorrhagic CNS event was counterbalanced by a decreased risk for an ischaemic CNS event. These were experienced by eight patients in the placebo group and by one patient with tifacogin.

\section{Defining a patient group for further study}

In a further attempt to elucidate the potential benefit of tifacogin in critically ill patients, the OPTIMIST study's clinical evaluation committee reviewed the information provided initially by participating centers and extracted findings only for those 496 patients who were judged to have had severe CAP. In these severe CAP patients overall, there was a trend toward improved survival in those who received tifacogin rather than placebo (mortality rate $27.9 \%$ versus $32.7 \%$; $P=0.25)$. This finding, albeit not statistically significant, was stronger when only those severe CAP patients with a microbiologically identified infection were included in the analysis. In this setting mortality with tifacogin was $27.1 \%$, as compared with $35.7 \%$ with placebo $(P=0.09)$.

A suggestion of benefit was also seen when only patients with severe CAP who did not receive heparin were included in the analysis; mortality with tifacogin was $29.1 \%$, as compared with $42.3 \%$ in the placebo group $(P=0.08)$. The effect became statistically significant when analysis was confined to patients with severe CAP with an identified infectious organism and no concomitant heparin exposure. In this group $(n=86)$, the $51.9 \%$ mortality rate among placebo patients was significantly higher than the $29.3 \%$ rate seen among tifacogin-treated patients $(P=0.02)$.

Among patients with severe CAP, a post hoc analysis of data from the OPTIMIST trial performed by a blinded clinical evaluation committee identified a trend toward benefit from tifacogin among patients in shock but not in those without shock [15]. The same pattern was seen for requirement for ventilation. Interestingly, a trend toward lower 28-day allcause mortality in patients receiving tifacogin was present across all quartiles of APACHE II score, suggesting a survival benefit independent of baseline severity and not restricted to severe CAP with multiple organ dysfunction.

These subgroup analyses were retrospective and, in some cases, involved small numbers of patients, with the attendant risk that prognostic variables were not well balanced across groups. However, there is encouraging consistency in the pattern that emerges. It appears that there is indeed potential benefit from tifacogin, provided that patients are not exposed to concomitant heparin, and there is a clear biological rationale to explain why there could be a negative interaction between the two agents. Furthermore, it appears that patients in whom a tifacogin benefit is most likely are those with severe CAP with a documented infection. These findings were influencing factors in the design of the ongoing Phase 3 CAPTIVATE study.

\section{CAPTIVATE (Community Acquired Pneumonia Tifacogin Intra Venous Administration Trial for Efficacy)}

The objective of this large, multicentre, placebo-controlled Phase 3 study (protocol number TFP561A2308) is to determine the safety and efficacy of tifacogin in adults with severe CAP admitted to the intensive care unit (ICU). The target accrual was 2,100 patients and enrollment was completed in July 2008. No data are currently available, and none of the investigator or steering committee members had access to the data. The databank should be closed by December 2008. 
Eligible patients have a clinical diagnosis of CAP based on the presence of at least two relevant signs (fever, tachypnea, leukocytosis, or hypoxemia) and radiographic findings of new pulmonary infiltrates within 24 hours of hospital admission. To fulfill the condition for severity, patients must either meet both the major Infectious Diseases Society of America/American Thoracic Society criteria (shock or requirement for ventilation) or have one major criterion or at least two minor ones. Because of the low but clinically relevant risk for bleeding, patients with a platelet count below $60,000 / \mathrm{ml}$ at baseline are excluded from the study.

In an attempt to document infection as thoroughly as possible, sputum and blood samples are collected during screening of the patient, along with urine for antigen determination. However, positive microbiology is not a requirement for study entry. Even so, the rate of microbiological documentation for patients accrued in Europe and Australia is running at $60 \%$, and the rate for the trial overall will probably reach $50 \%$.

In addition to receiving standard therapy in the ICU, patients are randomly assigned to one of three study arms: high-dose tifacogin $(0.075 \mathrm{mg} / \mathrm{kg}$ per hour), low-dose tifacogin $(0.025 \mathrm{mg} / \mathrm{kg}$ per hour), or matching placebo. Drug or placebo is administered by continuous intravenous infusion over 96 hours. Infusion must be commenced within 72 hours of hospital admission and within 36 hours of entry to the ICU. Prompt administration of tifacogin has been specified to ensure intervention takes place relatively early in the course of the disease process, before the development of disseminated intravascular coagulation and multiple organ failure.

The trial excludes patients requiring heparin therapy (either unfractionated or low-molecular-weight heparin), and use of heparin prophylaxis is not permitted during tifacogin infusion. During this 4-day period, thromboprevention is achieved with the use of intermittent compression devices. After the 4-day study drug infusion is completed, patients are allowed to receive heparin prophylaxis. Investigators have been asked to actively seek the development of any venous or arterial thrombotic event during and after study drug administration. To date, safety review by an external data monitoring committee has not identified any incidence of thromboembolic or bleeding events. The ongoing study also excludes patients receiving or expected to require treatment with drotrecogin alfa (activated protein C).

The primary end-point of the trial is all-cause mortality at 28-days in the intent-to-treat population. Comparisons will be made between patients randomly assigned to placebo, and tifacogin $0.025 \mathrm{mg} / \mathrm{kg}$ and tifacogin $0.075 \mathrm{mg} / \mathrm{kg}$. Analyses of tifacogin efficacy in relation to culture documentation of infection, baseline disease severity, and baseline coagulation status have been prespecified. To evaluate longer term outcome, data are being collected on survival at 90 days, 6 months, and 1 year.
To further contribute to our understanding of tifacogin's mechanism of action, the study is assessing changes in the coagulation biomarkers D-dimer, PF1.2, and thrombin-antithrombin complex. Pharmacoeconomic aspects of the trial include measures of resource utilization, the need for mechanical ventilation, and the duration of ICU and hospital stay.

\section{Discussion}

The preclinical data reviewed by other contributors to this supplement and the subgroup analyses of the initial Phase 3 OPTIMIST tifacogin trial described above provide a rationale for use of tifacogin in patients with severe CAP and hence for the ongoing CAPTIVATE study.

Subgroup analyses supporting the design of the CAPTIVATE trial have serious limitations because they considered small populations and therefore were statistically underpowered and potentially overestimated the effects of the studied drug. TFPI benefits in severe CAP have been retrospectively observed in patients with documented infection and in the absence of heparin prophylaxis. The outcome of the CAPTIVATE study will indicate whether this is reflected in improved outcomes in this large and important group of patients, whose mortality has not fallen appreciably since the 1950s. Also, the absence of heparin prophylaxis against deep vein thrombosis during the 4-day study drug infusion period may raise concerns. If the incidence of thrombotic events is increased in the placebo group compared with TFPI, then this may suggest that the outcome benefit is driven by the absence of heparin prophylaxis in this critically ill population. However, mechanical compression devices used in the study have been shown to be as effective as heparin prophylaxis in preventing peripheral thrombotic complications and should achieve adequate standard care.

The leading geographical region for enrollment has been Europe, followed by North America and Australia/New Zealand. Four unblinded safety evaluations have been conducted, along with two interim analyses. The fact that the study has been completed suggests that there have been no appreciable safety concerns and that there are indications of efficacy. The study has been successful in accruing its target population; retrospective scrutiny by the clinical evaluation committee has confirmed the CAP diagnosis in all but $6.5 \%$ of cases. Forty-seven per cent of patients to date have definite microbiological evidence of infection. Use of urine antigen tests, especially in European ICUs, has contributed to this encouragingly high level. This level of documented infection exceeds that seen in any comparable study.

It is increasingly accepted that severe CAP is a condition in which abnormal coagulation and an excessive host inflammatory response play an important role. Based on this understanding, a number of strategies for reducing morbidity and mortality are being pursued. Among them is use of glucocorticoid or other anti-inflammatory agents. At present, 
subgroups of patients in whom the benefits of such an intervention are likely to outweigh the risk for adverse effects cannot be identified. There appears to be a correlation between persistent inflammation (evident based on raised levels of IL-6, for example) and cardiovascular events occurring even during the weeks and months after pneumonia. This might justify further investigation into an anti-inflammatory strategy. However, stringent efforts to reduce early mortality through an intervention that has the potential to attenuate both the coagulopathy and the inflammatory processes associated with severe CAP continue to be a major priority.

\section{Competing interests}

PFL was engaged in the conduct of the CAPTIVATE study, is a member of the clinical evaluation committee of the CAPTIVATE study, and served as a consultant for Novartis and received speaker fees.

\section{Acknowledgements}

This article is based on a presentation made at a satellite symposium, 'Severe community-acquired pneumonia update: mortality, mechanisms and medical intervention', held on 21 April 2008 in Barcelona, Spain as part of the 18th European Congress of Clinical Microbiology and Infectious Diseases (ECCMID). It is published as part of Critical Care Volume 12 Supplement 6, 2008. The full contents of the supplement are available online at http://ccforum.com/supplements/12/S6

Publication of the supplement has been sponsored by Novartis.

\section{References}

1. Tapper $\mathrm{H}$, Herwald M: Modulation of hemostatic mechanisms in bacterial infectious diseases. Blood 2000, 96:2329-2337.

2. Creasey AA: New potential therapeutic modalities: tissue factor pathway inhibitor. Sepsis 1999, 3:173-182.

3. van der Pol T: Tissue factor as an initiator of coagulation and inflammation in the lung. Crit Care 2008, 12(Suppl 6):S3.

4. Welty-Wolf KE, Carraway MS, Miller DL, Ortel TL, Ezban M, Ghio AJ, Idell S, Piantadosi CA: Coagulation blockade prevents sepsis-induced respiratory and renal failure in baboons. $A m J$ Respir Crit Care Med 2001, 164:1988-1996.

5. Mandell LA, Wunderink RG, Anzueto A: Infectious Diseases Society of America/American Thoracic Society consensus guidelines on the management of community-acquired pneumonia in adults. Clin Infect Dis 2007, 44:S27-S72.

6. British Thoracic Society Standards of Care Committee: British Thoracic Society Guidelines for the management of community acquired pneumonia in adults. Thorax 2001, 56:1-64.

7. Matyal R, Mahmood F, Park KW: Tifacogin, recombinant tissue factor pathway inhibitor. Int Anesthesiol Clin 2005, 43:135144.

8. Creasey AA, Chang AC, Feigen L, Wun TC, Taylor FB, Hinshaw LB: Tissue factor pathway inhibitor reduces mortality from Escherichia coli septic shock. J Clin Invest 1993, 91:28502860.

9. Enkhbaatar $\mathrm{P}$, Okajima $\mathrm{K}$, Murukami $\mathrm{K}$, Uchiba $\mathrm{M}$, Okabe $\mathrm{H}$, Okabe K, Yamaguchi Y: Recombinant tissue factor pathway inhibitor reduces lipopolysaccharide-induced pulmonary vascular injury by inhibiting leukocyte activation. Am J Respir Crit Care Med 2000, 162:1752-1759.

10. Miller DL, Welty-Wolf K, Carraway MS, Ezban M, Ghio A, Suliman N, Piantadosi CA: Extrinsic coagulation blockade attenuates lung injury and proinflammatory cytokine release after intratracheal lipopolysaccharide. Am J Resp Cell Mol Biol 2002, 26: 650-658.

11. Park CT, Creasey AA, Wright SD: Tissue factor pathway inhibitor blocks cellular effects of endotoxin by binding to endotoxin and interfering with transfer to CD14. Blood 1997, 89:4268-4274.
12. Abraham E, Reinhart K, Svoboda P, Seibert A, Olthoff D, Dal Nogare A, Postier R, Hempelmann G, Butler T, Martin E, Zwingelstein C, Percell S, Shu V, Leighton A, Creasey AA: Assessment of the safety of recombinant tissue factor pathway inhibitor in patients with severe sepsis: a multicenter, randomized, placebo-controlled, single-blind, dose escalation study. Crit Care Med 2001, 29:2081-2089.

13. Abraham E, Reinhart K, Opal S, Demeyer I, Doig C, Rodriguez AL, Beale R, Svoboda P, Laterre PF, Simon S, Light B, Spapen H, Stone J, Seibert A, Peckelsen C, De Deyne C, Postier R, Pettila V, Artigas A, Percell SR, Shu V, Zwingelstein C, Tobias J, Poole L, Stolzenbach JC, Creasey AA; OPTIMIST Trial Study Group: Efficacy and safety of tifacogin (recombinant tissue factor inhibitor) in severe sepsis: a randomized controlled trial. JAMA 2003, 290:238-247.

14. Enjyoji $\mathrm{K}$, Miyata T, Kamikubo $\mathrm{Y}, \mathrm{Kato} \mathrm{H}$ : Effect of heparin on the inhibition of factor $\mathrm{Xa}$ by tissue factor pathway inhibitor: a segment, Gly 212-Phe243, of the third Kunitz domain is a heparin-binding site. Biochemistry 1995, 34:5725-5735.

15. Wunderink RG, Laterre PF, Larosa SP, Xie F, Poole L, Opal S: Severity of illness does not affect response to tifacogin in severe community-acquired pneumonia. AJRCCM 2006, supplement abstract issue. A 651 . 